\title{
Critical Thinking in Science: What Are the Basics?
}

\author{
Michael F. Shaughnessy ${ }^{*}$, Manuel Varela ${ }^{1} \&$ Zhiming Liu ${ }^{1}$ \\ ${ }^{1}$ Eastern New Mexico University, Portales, New Mexico \\ * Michael F. Shaughnessy, E-mail: Michael.Shaughnessy@enmu.edu
}

Received: October 1, 2017

doi:10.22158/wjer.v4n4p585
Accepted: October 20, 2017 Online Published: November 2, 2017

URL: http://dx.doi.org/10.22158/wjer.v4n4p585

\begin{abstract}
This paper reviews some of the most critical issues in science in terms of scientific thinking, and reasoning. Many students arrive at college poorly prepared to function in the typical science class and encounter a good deal of difficulty. This paper provides some general information regarding critical thinking skills and delves into some specific realms that need investigation and formative evaluation.
\end{abstract}

\section{Keywords}

critical thinking, higher order thinking, science, scientific reasoning, hypothesis testing

\section{Introduction}

All too often, students arrive at the college freshman level lacking critical thinking skills. In no realm is this more apparent than in the sciences. This paper will examine and explore the reasons for this deficiency and discuss needed on-going remediation efforts.

It is a scenario all too often encountered. College freshmen arrive lacking basic terminology, basic understanding of processes and procedures, and lacking expertise with the basic scientific method. The reasons for this are many and varied and probably worthy of empirical exploration. In any event, this paper will provide some basic elements needed for adequate critical thinking procedures and processes in the realm of science.

While science is often learned through observation, language and vocabulary is often foundational. Some basic terminology follows. Many students do not understand the language, the concepts; the importance of these words, or confuses the terms and have forgotten some of the most salient, critical terms. These shall be reviewed below:

a) Variable - any item of interest, any factor of interest, any specific thing or element that could have an impact on the outcome of an experiment. It is important in scientific thinking to consider all variables that could have an impact on the experiment, or the outcome of the experiment.

b) Independent Variable - this is the variable that is going to be manipulated. This is the variable that might be increased or decreased as the experiment continues, or a specific factor that needs to be examined to see its impact on another variable that will be examined or measured in some way, shape, 
manner, fashion or form. It needs to be clearly understood that in some instances, this is your "treatment". In science and in other areas of research, the "treatment" could consist of a number of tings - such as the passage of time, as well as vitamins, nutrients, proteins, minerals and the like.

c) Dependent Variable - This is the outcome or something to be measured to ascertain the impact of the independent variable. This variable needs to be measured precisely, exactly, specifically, and concisely in order to determine if there is some impact of the independent variable on this dependent variable. Many beginning scientists have trouble or difficulty clearly indicating how the dependent variable is going to be measured, evaluated or assessed - either formally or informally.

d) Hypothesis-This is the scientist's hunch or guess, or impression about the impact of an independent variable on another dependent variable. Often this is couched in very specific language such as a Main hypothesis or alternative hypothesis. On occasion, we have a hypothesis that something will increase or decrease — or that there will be no difference whatsoever.

e) Alternative Hypothesis-We have a "back up hypothesis" so to speak on the off chance that the independent variable will not cause any change in the dependent variable. So, we often write out an alternative hypothesis, keeping in mind, that the strength of our treatment or independent variable may not bring about any change or impact it to a statistically significant degree.

f) Control group-In some experiments, we have a group that does not receive the independent variable- or they could receive what is often terms a "placebo" or inert sugar pill. This is to determine if the passage of time perhaps caused the increase or decrease or change in the dependent variable, or to determine if there is some other factor or variable at work in our design.

g) Control-By control, we mean that we are trying to keep conditions as standardized as possible. The temperature of the room for example, the amount of medication or vitamin being administered, or the amount of sunlight in certain plant experiments may also need to be controlled as a variable of interest.

h) Environment-In some scientific experiments we have a certain environment that needs to be kept sterile, and the room temperature or amount of sunlight or even air needs to be meticulously controlled in the environment in which the study is being conducted.

i) Time-The measurement of time is of particular importance in many experiments. The amount of time that the subjects will be receiving the treatment or the independent variable is of paramount importance. The amount of time it takes a subject to perform a certain physical task and the amount of time that the scientist projects for the experiment is important to note. We need to keep meticulous attention to these details as time can be a salient, relevant variable.

j) Treatment-In medical and educational research, there are attempts to ascertain the treatment effects of a certain factor-In medicine, a new anti-biotic may yield a better treatment result than other older anti-biotics. In education, a new approach to reading could bring about a statistically significant improvement in reading rate, comprehension or other aspect of reading. In some realms, it is difficult to quantify "treatment" as counseling for example could be seen as a form of "treatment", and it is difficult to ferret out or separate what exactly is bringing about emotional or cognitive or behavioral 
change.

k) Multiple Variables - Sadly, this is a complex world and quite often we need to examine more than one variable as possibly operative in any experiment. Scientists would do well not to think univariately in a multi-variate world. As we begin to explore more and more variables, we see that there could be other factors or variables that are operative in an experiment.

k) Theory-While there are certain laws in science, there are many theories that need to be examined, explored and verified. The law of gravity is one that holds true on earth, but may not be operative on Mars.

1) Statistics - In order to mathematically and empirically prove a hypothesis, based on a theory, we have to conduct a controlled experiment, in which we examine numbers and utilize the realm of statistics to prove or disprove a theory or a hypothesis. There are various forms of statistics-parametric and non-parametric and various other realms of statistics, and it is incumbent on the scientist to utilize the correct statistics to ascertain if the treatment was in fact effective and to ascertain if their hypothesis was accepted or rejected.

Equipment-On most college campuses, there are several "basic" instruments of science that need to be utilized for the learning of science. While most students encounter these in high school biology or chemistry, these are the basic tools of the scientist.

The Microscope-The microscope enables students to perceive and observe what is not available to the naked eye. The microscope allows students to visually scan and examine various elements and things that may be written about in textbooks- but are not seen in operation so to speak.

The Petri Dish-One of the most basic, yet one of the most valuable components of a science lab, the Petri dish serves as a veritable environment for experimentation, observation and preservation on occasion of samples.

Test Tubes-Although much learning has been put online and on Power Points and PREZI, the real touch of a test tube remains a staple of laboratory experimentation. The student can observe chemical reactions, watch things change and observe interactions

Bunsen Burner-Perhaps anachronistic, the Bunsen burner is perhaps used in some places, by some scientists with admirable results for good teaching and experimentation in labs. Heat, as a variable, as a factor needs to be continually examined and explored in terms of chemical reactions.

Past Investigations-A good literature review can provide a wealth of information for the serious scientist and save a good deal of time effort and energy. The Internet or World Wide Web allows scientists now availability to a number of journal articles in their field of interest and a cursory review or skimming of the abstract will allow the critically thinking scientist to determine if their study has already been conducted by some other fact seeking, fact checking scientist.

Machi and McEvoy (2016) have written an excellent book on the literature review process. For many students, they have not been adequately trained to write a good, cogent, coherent literature review and this book serves as a good starting place for them. Obviously, reading other good literature reviews is 
another important part of the process, and instructors should make good literature reviews available to students as templates or models of exemplary literature review, synthesis and integration.

All too often, pupils lack the foundational knowledge of prior scientific explorations. Certainly, most student know about the laws of gravity. However, the construct of combustion may not have been specifically discussed. Photosynthesis may have been cursorily reviewed but an in-depth understanding as to what occurs at the molecular level has not been forthcoming. The people-the scientists, the researchers, the leaders in the field that have investigated certain domains should be delineated so as to provide a comprehensive overview of the realm of science in which they are working.

\section{Thinking Precisely as a Part of Critical Thinking}

High school students typically arrive at college lacking a certain precision of thinking. They tend to make vague, nebulous, global, imprecise statements and generalities or engage in what is often referred to as: "Shot gun empiricism".

Part of their training should be to assist them in focusing and utilizing very exact, precise, terms and terminology. Students need to read, write, speak and think using scientific terms and modalities.

Time is an issue in terms of training pupils to speak, think, and write as scientists or at least using scientific terms and terminology. Time must be allocated so that students can in fact reflect on what they have to say, and how they are going to write and communicate in a professional scientific manner. The process need not be arduous, but time must be devoted to this area.

If students have had a course in statistics, they may be most comfortable discussing .05 or .01 level of confidence. Basically, these confidence levels are set before an experiment begins so as to ensure fidelity of treatment and ethical behavior. If the statistical level is set at .05, then there is a 95 percent chance that there was in fact, a treatment effect—or that the independent variable did have some impact on the dependent variable. Medical research often sets the confidence level at .01, indicating that they are 99 percent confident in their results and that there is only a 1 percent chance out of 100 that they could be wrong or that the results could be due to luck, chance, fate or some other factor.

The issue of operational definition is an important one. This is a concept that is often lost on the murky thinking of college students. The idea behind an "operational definition" is to allow scientists to communicate and facilitate replication. An "operational definition" is one that allows scientists to communicate and understand each other and to "have a handle" on what other people are investigating. Sometimes a broad general construct such as "alcoholism" is too broad and general, and we need to specify, how we are going to identify such individuals and how we are going to diagnose or label these individuals. 


\section{The Laboratory As a Critical Part of Scientific Thinking}

Many feel that it is essential for science students to perform science experiments in the labs. In this way, they assimilate the procedures, observe processes and make at least preliminary inferences.

Therefore, the following five skills are important to them when they obtain information from their experiments:

1) Interpretation: students must have the ability to understand the information they have received.

This may include observational inferences, this may include procedural inferences and this may include results and the summarizing of results.

2) Evaluation: they should be able to evaluate the credibility of the information they have received. Herein, they have to think logically, rationally, reasonably, scientifically, empirically and vigorously. And this is something that they may not have engaged in during past years or past semesters.

3) Analysis: they need to assess the logical interrelationship among the facts from various experiments. The emphasis here is on facts, data, information and conclusions drawn from specific facts and information. The student must analyze and perhaps even write down their analytic procedures for the instructor or a colleague to review.

4) Inference: they need to have the ability to perceive any implications from the information. While some would refer to this as a conclusion, it is predicated on the hypothesis and the results and the observations that have occurred. There is obviously no 100 percent sure conclusion that can be reached unilaterally, but inferences can be drawn and summarized from the data.

5) Explanation: they need to develop the ability to restate the information and present it to others in an understandable way. This skill overlaps with communication and writing. The student needs to be able to clearly, and succinctly write in a scientific manner and communicate and explain what exactly has transpired.

Three key words are relevant here. The three words are: face to face. The lab provides an exceptional opportunity for instructors to gauge student understanding of material, processes and procedures. Further the face to face exploration assists in comprehension of material and understanding of complex intricate constructs, terms and details.

\section{Exploratory Thinking}

Sternberg and Davison (2007) have discussed insight in the gifted but their ideas are most applicable and relevant to scientists who are on the cutting edge of research and have the time to devote to their research endeavors.

Sternberg and Davison (2007) have put forth a sub-theory of intellectual giftedness which is most applicable to scientific thinking and reasoning and which is applicable to scientists insight skills, learning and ability to formulate new hypotheses and relevant experiments. These Insight skills are indicated to be of three different kinds and will be discussed in depth.

The first is termed "selective encoding" and while some may disagree with our definition and 
interpretation, this is the process by which salient, germane information is learned, and in a sense focused on. Excellent scientists determine what is relevant, and what is relevant in much the same way that good readers determine what is most important in any given paragraph and in any given chapter and in any given book. In a sense, the good scientist is also able to determine relevant information, and relevant feedback from irrelevant information and tangential, superfluous information.

The second methodology of processing is called "selective combination". The scientist that excels is able to sort through a lot of rich, robust, relevant information and then combine it in new, novel and perhaps divergent ways, or ways that will lead us to new hypotheses or perhaps to new vistas of research. The good researchers and scientists are combining variables that are relevant. They are combining procedures that are applicable and which have shown promise in the past. These scholars are the ones that take from various past researchers and past published works and have synthesized and integrated the ideas of some of them most productive individuals doing research for decades. They are more productive in their fields of endeavor due to these skills and combinations. There is an "a ha" effect much the same as past researchers have been enlightened by the falling of an apple or something that occurred to them in a dream or in a bathtub.

The last skill is selective comparison and in this information processing model, new information is linked to old or established information in a new or divergent or flexible or novel way too old, previously established published or well-known information.

These skills are difficult to quantify and difficult to replicate. We can study the theory of these ideasbut it is more difficult to teach these patterns of thinking. Only through ongoing experimentation, and on-going supervision, and discussion can these ideas really be integrated into the mind of novice scientists.

\subsection{In Depth Thinking}

The novice science student thinks differently than the more sophisticated "expert" researcher, and the expert researcher has obviously more statistical acumen at their disposal than the novice. The expert is aware of various different methodologies and the correct machinery that may expedite and facilitate their research.

Kuhn, Iordanou, Pease and Wirkala (2008) have attempted to explore the skills and cognitive capacities that need to be developed in order to achieve mature, robust, skilled scientific thinking.

Kuhn et al. have very clearly indicated that "scientific thinking is multivariate reasoning". In other words, they emphasize that scientists should not be thinking uni-variately in a multivariate world. They secondarily focus on the idea that scientific thinking reflects a true understanding of the nature of science. They do not believe in superficial thinking, but in-depth reasoning, critical thinking and higher order thinking about variables and the control of variables. Lastly, Kuhn and her colleagues argue that scientific thinking should include argumentation - that a true scientist must be ready, willing and able to debate, discuss and argue their point of view and be prepared to confront colleagues that may not necessarily agree with their premises or ideas. 
Kuhn (2007) in an earlier piece has argued that control of variables is not the only challenge confronting scientists and those engaged in the experimental method. Certainly, it is one part of the entire paradigm, but not the only part. In addition, variables are often neglected which are relevant, salient and germane to the study, but often ignored, or given short shrift.

Varela, Lutnesky and Osgood (2005) have assessed the ability of students to identify key aspects of published papers and to measure critical thinking skills used to evaluate scientific validity, using a primary trait analysis scale.

Obviously, time is a factor and ample time for reflection, perhaps discussion and review of relevant, salient factors is important. Today's students often need to allocate an adequate amount of time to simply study, review, examine and explore what they are doing and what they are trying to attempt.

\subsection{Key Critical Thinking Abilities}

The proficient, experienced scientist has the following abilities in terms of their cognitive structures and cognitive abilities. They have the:

1) Ability to make logical conclusions based on evidence.

They think logically, rationally, reasonably and process information quite systematically and look for the data, the evidence, the proof and the final numbers (if there are any).

2) Ability to logically apply methods to evaluate hypotheses.

In a sense, these scientists are juggling two disparate skills. They know exactly what type of methodology to use in order to procure the answer to their questions, and they are able to both verbally and in writing, articulate their specific hypothesis that is testable, reasonable, rational, logical and systematic, and also worthy of investigation.

3) Ability to interpret, analyze and evaluate new information.

The expert scientist is able to interpret data-from beginning to middle to end of the experiment, analyze the results (in light of past literature, and in light of the results procured and then are able to integrate and analyze new information and new theories, and perhaps even integrate this new information into their constructs and on-going theory development).

4) Simultaneous identification of problems and their corresponding effective solutions.

As expert scientists build on solid foundations of past research and build on past evidence and research. They are able to simultaneously identify new challenging problems and concerns and then hypothesize perhaps plausible, effective solutions, or at the very least, new experimentation that would shed light on the problem and present at least tentative solutions to the problem.

5) Ability to logically formulate a hypothesis based on previous knowledge.

At the foundation of all scientific research is the need to have a clear hypothesis, based on previous research, based on the literature, and based on past results (which may or may not have been shared by colleagues in print or in presentation modality. Previous knowledge, or what is often called "world knowledge" or "general information" is imperative here. The expert scientist has a rich robust storehouse of background knowledge and information about their field, and are able to sift through past 
research to find the relevant, salient, appropriate domains to investigate or at least explore.

6) Ability to identify the importance of a scientific field.

The master scientist looks carefully at the field and is able to discern where the field is going at that particular point in time. Some scientists simply keep up with the literature, while others attend conferences to ameliorate their knowledge base and find out what is transpiring in the field, while others simply have an uncanny knack for seeing where the field is going- be it biology, chemistry, physics, or whatever. They realize the importance of their field and how it fits into the big picture.

7) Ability to analyze and objectively evaluate data for determination of its validity.

Objectivity, neutrality, and the ability to withhold judgment until the appropriate time is imperative for science, and scientists in general. The master scientists knows how to analyze data as well as disaggregate the data when appropriate. The key words of validity, and reliability are scorched into their minds as key constructs of the scientific method.

8) Ability to recall and understand knowledge.

Since the field is changing ever so rapidly, the master scientist must be able to understand and grasp new concepts, new ideas, new constructs, and new knowledge- or at least be able to ascertain where to get the information so as to be able to understand new discoveries.

9) Ability to classify, summarize, and compare theories.

Piaget has written extensively about the cognitive processes involved in classification. It is a cognitive processing skills that some have, some develop and some struggle with on an ongoing basis. Some scientists rely on external aids (graphs, charts, pictures) and others seem to automatically process classifications and relevant information.

10) Ability to understand methods and to apply them to evaluate hypotheses.

There needs to be a link between methodology and the hypothesis. It is not clear if this connection is always made or if it is after the fact, or if one comes immediately before the other. Some scientists adhere to one methodology in which they were trained and do not differ from it during their adult lives. Other scientists are willing to pick and choose and ascertain the most appropriate methodology for their investigations.

\subsection{Metacognition}

Although somewhat beyond the scope of this introductory paper, college and graduate students may need to critically reflect on what they are thinking about, their inductive and deductive reasoning skills, their own scientific reasoning skills, and areas and domains that they may have neglected. An excellent book on Metacognition has been edited by Vennemann, Shaughnessy and Kleyn Kennedy (2008).

\section{Summary and Conclusions}

This paper has cursorily attempted to review the realm of critical thinking in terms of science, science experimentation and the thought processes that go into formulate, conducting, analyzing and synthesizing and integrating the data that emanates from any scientific experiment. Some experiments 
are formal, and conducted in a highly structured manner, while others are more informal and conducted perhaps in a cursory manner. The teaching of scientific thinking, reasoning, critical thinking and multi-variate thinking is a realm that needs further exploration and analysis as well as critical discussion in the future.

\section{References}

Baron, J. B., \& Sternberg, R. J. (1987). Teaching thinking skills: Theory and practice. NY.

Freeman, W. H., \& Co. Fascione, P. A. (2015). Critical thinking: What it is and why it counts Measured Reasons. Hermosa Beach, CA.

Kuhn, D. (2007). Reasoning about multiple variables: Control of variables is not the only challenge. Science Education, 91(5), 710-716. https://doi.org/10.1002/sce.20214

Kuhn, D., Iordanou, K, Pease, M., \& Wirkala, C. (2008). Beyond control of variables: What needs to develop to achieve skilled scientific thinking? Cognitive Development, 23, 435-451.

Machi, L. A., \& McEvoy, B. T. (2016). The Literature review: Six Steps to Success Corwin. CA.

Shaughnessy, M. F. (Ed.). (2014). Critical thinking and higher order thinking: A Current Perspective NY Hauppauge. NOVA.

Sternberg, R. J., \& Davison, J. E. (2007). Insight in the gifted. Educational Psychologist, 18(1), 51-57. https://doi.org/10.1080/00461528309529261

Stewart, O. G., \& Jordan, M. E. (2016). Some explanation here: A case study of learning opportunities and tensions in an informal science learning environment. Instructional Science.

Varela, M. F., Lutnesky, M. M. F., \& Osgood, M. P. (2005). Assessment of Student Skills for Critiquing Published Primary Scientific Literature Using a Primary Trait Analysis Scale. Microbiology Education, 6, 20-27. https://doi.org/10.1128/154288105X14285806518972

Vennemann, M., Shaughnessy, M., \& Kleyn, K. C. (2008). Meta Cognition: A Recent Review of Research, Theories and Perspectives. Hauppage, New York Nova Publishing. 